\title{
BMJ Open Study protocol for a prospective process evaluation of a culturally secure rehabilitation programme for Aboriginal Australians after brain injury: the Healing Right Way project
}

\author{
Rachel Skoss (1) , ${ }^{1,2,3}$ Jane White, ${ }^{4}$ Mandy J Stanley (1) , ${ }^{4}$ Melanie Robinson, ${ }^{5}$ \\ Sandra Thompson, ${ }^{6}$ Elizabeth Armstrong (D) , ${ }^{7}$ Judith M Katzenellenbogen (D) ${ }^{3}$
}

To cite: Skoss R, White J, Stanley MJ, et al. Study protocol for a prospective process evaluation of a culturally secure rehabilitation programme for Aboriginal Australians after brain injury: the Healing Right Way project. BMJ Open 2021;11:e046042. doi:10.1136/ bmjopen-2020-046042

- Prepublication history for this paper is available online. To view these files, please visit the journal online (http://dx.doi. org/10.1136/bmjopen-2020046042).

Received 23 0ctober 2020 Accepted 05 July 2021

Check for updates

(C) Author(s) (or their employer(s)) 2021. Re-use permitted under CC BY-NC. No commercial re-use. See rights and permissions. Published by BMJ.

For numbered affiliations see end of article.

Correspondence to Dr Rachel Skoss; rachel.skoss@nd.edu.au

\section{ABSTRACT}

Introduction Aboriginal and Torres Strait Islander (hereafter respectfully referred to as Aboriginal) people are Australia's First Peoples, having the longest continuous culture in the world and deep spiritual connections with ancestral land. Improvements in their health and wellbeing is a major policy goal of Australian governments, as the legacy of colonisation and disruption of cultural practices contribute to major health challenges. Lack of culturally secure services impacts participation of Aboriginal people in health services. Aboriginal people with a brain injury typically experience poor access to rehabilitation and support following hospital discharge. 'Healing Right Way' (HRW) is a randomised control trial aiming to improve access to interdisciplinary and culturally secure rehabilitation services for Aboriginal people after brain injury in Western Australia, improve health outcomes and provide the first best practice model. This protocol is for the process evaluation of the HRW trial.

Methods and analysis A prospective mixed methods process evaluation will use the Consolidated Framework for Implementation Research to evaluate implementation and intervention processes involved in HRW. Data collection includes qualitative and quantitative data from all sites during control and intervention phases, relating to three categories: (1) implementation of trial processes; (2) cultural security training; and (3) Aboriginal Brain Injury Coordinator role. Additional data elements collected from HRW will support the process evaluation regarding fidelity and intervention integrity. Iterative cross-sectional and Iongitudinal data synthesis will support the implementation of HRW, interpretation of findings and inform future development and implementation of culturally secure interventions for Aboriginal people.

Ethics and dissemination This process evaluation was reviewed by The University of Western Australia Human Research Ethics Committee (RA/4/20/4952). Evaluation findings will be disseminated via academic mechanisms, seminars at trial sites, regional Aboriginal health forums, peak bodies for Aboriginal health organisations and the Australian Indigenous HealthlnfoNet (https://healthinfonet. ecu.edu.au/).

Trial registration number ACTRN12618000139279.

\section{Strengths and limitations of this study}

- This is a culturally secure, context-sensitive prospective process evaluation of a complex health service intervention to address an important gap in service delivery for Aboriginal people.

- The evaluation uses the Consolidated Framework for Implementation Research using robust mixed methodology.

- A strength of this evaluation is the iterative crosssectional analysis and synthesis of data to inform the implementation of the intervention, together with the longitudinal analysis and synthesis of data to support interpretation of findings.

- A limitation will be the possibility of incomplete quantitative data relating to staff turnover, changes in site operations and completion of online surveys at each site where cultural security training is conducted. Such data will be challenging to consistently monitor and collect; however, mechanisms are in place to extract relevant data from study documentation.

\section{INTRODUCTION}

In real world settings, implementation failure can threaten clinical trials. How interventions are operationalised and implemented can seriously compromise their intended outcomes. There is increasing recognition that randomised controlled trials (RCT), especially those determining the effect of complex interventions, require detailed process evaluation to ensure that trial results can be interpreted and translated correctly. This applies particularly to interventions targeting long-term conditions necessitating multidisciplinary care. The Medical Research Council has recommended that process evaluation within trials 'be used to assess fidelity and quality of implementation, clarify causal 
mechanisms and identify contextual factors associated with variation in outcomes'. ${ }^{1}$

Aboriginal and Torres Strait Islander (hereafter respectfully referred to as Aboriginal) people are Australia's First Peoples, having the longest continuous culture in the world and deep spiritual connections with ancestral land. In contemporary times, Aboriginal Australians comprise $3.3 \%$ of the population and have a younger age structure than other Australians (median age 23 vs 38 years). ${ }^{2}$ Improving the health and well-being of Aboriginal Australians is a major policy goal of the Australian government, with the legacy of colonisation and ongoing disruption of cultural and linguistic practices contributing to social, emotional, financial and health challenges. ${ }^{3}$

The nature of Aboriginal peoples' experiences and interactions with the health system is increasingly recognised as a major determinant of health/disability outcomes. System barriers, especially the lack of culturally secure services, ${ }^{4-6}$ have been shown to impact on the accessibility of health services for Aboriginal people..$^{7-9}$ Cultural security is an attribute of services, where institutions and their staff have awareness of Aboriginal cultural values, practices and world views and act on this knowledge to address patient needs. ${ }^{10}$ This requires ongoing examination and questioning of cultural assumptions and biases at both an individual and institutional level. Culturally secure services have policies and procedures to ensure appropriate actions are applied systematically across the service, so as not to compromise the legitimate cultural rights, values and expectations of Aboriginal people. ${ }^{11}$ Achieving and maintaining cultural security is necessary for improving Aboriginal people's access to and the care they receive from services.

Acquired brain injury (ABI) often results in motor, communication, sensory and cognitive impairments that can adversely affect quality of life in the long term, including employment prospects, family relationships, social participation and mental health. ${ }^{12} 13$ The mixed methods Missing Voices Project ${ }^{14}$ reported substantial differentials in the burden of stroke and traumatic brain injury (TBI) between the Aboriginal and non-Aboriginal populations of Western Australia (WA), contributing to higher rates of ABI experienced by Aboriginal people. ${ }^{15} 16$ Importantly, Missing Voices identified challenges in acute hospital care for Aboriginal people with stroke and TBI, and poor access to rehabilitation and support following hospital discharge. Many non-Aboriginal clinicians reported that they did not possess the skill and knowledge to provide satisfactory rehabilitation services to Aboriginal patients with brain injuries. Aboriginal participants reported communication breakdowns with hospital staff, a lack of acknowledgement and accommodation of cultural protocols and practices, and a need for more practical support during recovery from brain injury. ${ }^{56}$ Quantitative and qualitative brain injury research around Australia provides further evidence of the need to provide culturally secure rehabilitation services for Aboriginal people and families. ${ }^{12} 13$ 17-19 The current Clinical Guidelines for Stroke Management ${ }^{20}$ acknowledge that Aboriginal people have additional needs that demand special attention and resources yet provide no specific guidelines for practice in stroke or TBI care for these patients, and rehabilitation programmes for brain injury are currently not meeting the needs of Aboriginal people.

Consequently, funds from the National Health and Medical Research Council support the first-ever clinical trial-Healing Right Way (HRW) - which aims to improve access to interdisciplinary and culturally secure rehabilitation services for Aboriginal people with ABI, improve health outcomes and provide a robust best practice model for this population, supported by evidence (box 1). Such a model needs to acknowledge the diversity of people involved across the country and the need for incorporation of local protocols, knowledge and practices. The HRW trial is informed by the findings of the Missing Voices Project and our team's earlier work that focused on the experiences of Aboriginal people with brain injury and their families and related health professionals across WA and their recommendations for future services. ${ }^{5614}$ The HRW team uses an Aboriginal research framework ${ }^{21} 22$ incorporating principles based on Indigenous Standpoint Theory, ${ }^{23}{ }^{24}$ where Aboriginal peoples' experiences, recommendations and leadership in the research process are central and which has been applied to disability in an Aboriginal context. ${ }^{25-27}$ This research has provided insights and directions forward, including regarding Aboriginal peoples' concepts of disability and impairment, participation in disability services and Aboriginal workforce development. Accordingly, the HRW team consists of Aboriginal and non-Aboriginal researchers who work collaboratively, recognising varying personal experiences and backgrounds from which we come that can influence/bias the way we approach our research at all levels. The team has partnerships with Aboriginal Community Controlled Health Organisations and other government and non-governmental organisations to strengthen the translational aspects of the study and ensure Aboriginal perspectives are embedded. The project has established study sites across WA ( 2.6million square kilometres) and across language groups. Two-way learning and consultation at each site will occur throughout the project, which is guided by an Aboriginal Reference Group (see main study protocol in a forthcoming edition of this journal ${ }^{28}$ for details).

However, health interventions often do not show significant changes in outcomes for Aboriginal people, frequently due to implementation failure. Understanding the challenges and opportunities in implementing programmes and acting on the knowledge gained has the potential to substantially improve the success of health interventions and thereby the health of Aboriginal Australians. Given the complexity ${ }^{29}$ of the HRW intervention, a process evaluation (figure 1) was designed to support trial implementation, facilitate interpretation of results and ensure that knowledge gained during and from the 
Box 1 Brief outline of the parent Healing Right Way (HRW) trial

\section{Study design}

Healing Right Way is a randomised controlled trial focused on enhancing rehabilitation services and quality of life for Aboriginal Australians experiencing impairments following stroke and TBI and may be considered a complex intervention. ${ }^{29}$ It employs an Aboriginal Research framework. ${ }^{21}$ The stepped wedge cluster trial design involves sequential but random rollout of the intervention to four metropolitan and four regional Western Australian hospitals. Control (non-intervention) data collection for each site will be for a minimum of 6 months, while all sites receive the intervention for a minimum of 12 months. The intervention will be added to one metropolitan and one regional site per intervention step.

\section{Recruitment}

Aboriginal people, over 18 years, admitted to hospital for acquired brain injury $(A B I)$ resulting from stroke or traumatic brain injury, will be recruited from 2018 to 2021.

\section{The two-pronged intervention comprises}

1. Cultural security training encompassing aspects of care specific to $\mathrm{ABI}$ patients targeting hospital staff in both face-to-face group and online formats.

2. Training and employment of region-based Aboriginal Brain Injury Coordinators $(A B I C)$ to support Aboriginal people with $A B I$ for 6 months postinjury.

\section{Outcomes}

The primary outcome measure is quality of life as measured on the EuroQ0L-5D-3L* at 6 months postinjury.

Secondary outcome measures relate to severity of disability (modified Rankin Scale†), functional independence (Functional Independence Measure -FIM $\ddagger$ ), burden of care (Carer Strain Index§), and clinical service provision (process of care indicators).

\section{Data collection}

Trained assessors will collect baseline data within 6 weeks of injury, and follow-up data within 12 weeks and 26 weeks postinjury.

\section{Patient and public participation}

Aboriginal participation includes partnership with Aboriginal-run organisations, Aboriginal reference group and regular meetings with community stakeholders, all of whom provided feedback on the design and implementation of the intervention. Aboriginal participants and ABICs (Aboriginal employees) will provide feedback on the intervention. The reference group will assist in interpretation of results and development of the translation plan.

*EuroQ0L-5D-3L (The EuroQol Group. EuroQol-a new facility for the measurement of health-related quality of life. Health Policy 1990;16:199-208). † Modified Rankin Scale (Van Swieten J, Koudstaal P, Visser M, et al. Interobserver agreement for the assessment of handicap in stroke patients. Stroke 1988;19:604-07).

fFunctional Independence Measure (Keith RA, Granger CV, Hamilton BB, et al. The functional independence measure: a new tool for rehabilitation. Adv Clin Rehabil 1987;1:6-18)

$\S$ Carer Strain Index (Thornton M, Travis SS. Analysis of the reliability of the modified caregiver strain index. The Journals of Gerontology Series B: Psychological Sciences and Social Sciences 2003;58:S127-32).

trial is embedded in services, disseminated and translated appropriately to maximise lessons learnt. ${ }^{1}$

This paper describes the protocol for the process evaluation, embedded within the stepped-wedge HRW trial (figure 1). The aim of the process evaluation is to determine whether the research processes and interventions were implemented as planned and investigate contextual factors present during the different trial phases (baseline and the implementation of each phase/wedge of the intervention) of HRW that may have influenced implementation and trial outcomes. Specific key objectives are to provide:

1. A process analysis running in parallel to the trial to inform and refine the HRW intervention within the confines of an RCT.

2. Retrospective analysis to support interpretation of the outcomes of HRW and allow for explanation of the study results. ${ }^{30}$

3. Reflection on lessons learnt and the tools developed to inform future development and implementation of culturally secure interventions for Aboriginal people.

\section{METHODS AND ANALYSES \\ Study design}

This mixed methods study is nested within the HRW trial. The study will use prospectively collected qualitative and quantitative information and be characterised by an iterative process in which the data informs discussion, assists in interpreting the results of the trial and ultimately the conclusions.

\section{Conceptual framework for the process evaluation}

The process evaluation will use the Consolidated Framework for Implementation Research (CFIR), ${ }^{31}$ enabling the recording of contextual detail throughout the implementation and conduct of HRW. The CFIR considers key process components outlined by Linnan and Steckler, ${ }^{32}$ providing a pragmatic approach to evaluation in a complex and often disorganised real-world setting, where a range of factors can act, interact and influence an intervention. Such is the case in HRW where the intervention occurs in multiple health settings of varying size and varying resources. ${ }^{31}$ This framework ${ }^{31}$ has been used in other health-related ${ }^{33}$ and Aboriginal contexts ${ }^{34}$ and is useful to guide rapid cycle evaluation to systematically identify where adjustments and refinements can be made during implementation, meeting our objective to inform/refine the HRW intervention.

The CFIR offers scope for a developmental evaluation approach while maintaining a focus on the intended goals $^{35}$ to improve health outcomes for Aboriginal people with brain injury. This approach allows the evaluation team to take an emergent perspective and respond to unpredictable and evolving environments as HRW progresses $^{35}$ and identify and quantify unexpected and unintentional outcomes. ${ }^{30}$ It is important to consider the extent to which an intervention is implemented as planned, as real-world context can affect the implementation, ${ }^{36}$ for example, the varying contexts present in different healthcare settings. ${ }^{37}$ Variation in implementation of HRW can reasonably be expected via local 


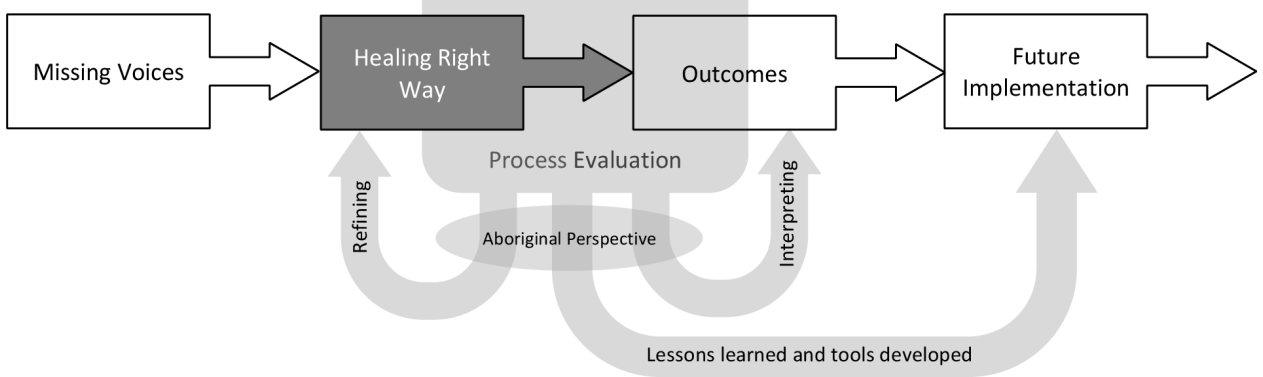

Figure 1 Schematic of the role of the process evaluation in HRW. HRW, Healing Right Way.

adaptation, ${ }^{35}$ given the geographic diversity of health sites together with the diversity of the communities they serve. As shown in figure 2, the CFIR allows consideration of this diversity (eg, the outer and inner settings and individuals involved), and their potential influence on the intervention (characteristics of intervention) and the trial processes themselves (implementation process). This can then support the planning of more widespread implementation ${ }^{30}$ and provide insight for planning and design of services for similar cohorts/contexts.

Cultural security of the evaluation itself will be guided by the Aboriginal members of the evaluation and HRW research teams and the feedback of the Aboriginal Reference Group. This group consists of Aboriginal clinicians, Aboriginal people who have had a stroke, family members and community elders. Specifically, data collection methodologies and analysis will be informed by Aboriginal team members. ${ }^{38}$

\section{Data collection and measurement}

The process evaluation will use a mixed methods approach to data collection, collecting qualitative and quantitative data from all sites during the control and intervention phases of the study ${ }^{39}$ We will collect three categories of data related to: (1) implementation of the trial processes;
(2) cultural security training intervention and (3) ABIC service as an intervention. Additional data elements will be collected from HRW that will support the process evaluation with respect to fidelity and intervention integrity. All interview participants will provide written consent.

\section{Trial processes}

A 'project log' will capture the evolving nature of HRW due to implementation and contextual factors. This project log is an electronic database containing a summary of events/issues related to the day-to-day planning and implementation of HRW, not otherwise captured by meeting minutes. Details of these events will be provided by the trial's chief investigator and project manager, usually by forwarding electronic working notes made on mobile devices, and through independent observation of ongoing processes by a member of the process evaluation team. Once captured, the stored events can be grouped and sorted by date and site, who was involved, relevance to specific processes and actions or resolutions that may eventuate.

During the control phase, and in each phase of the stepped-wedge design, semistructured interviews will be held with key programme staff, including the chief investigator, project manager, data operations manager

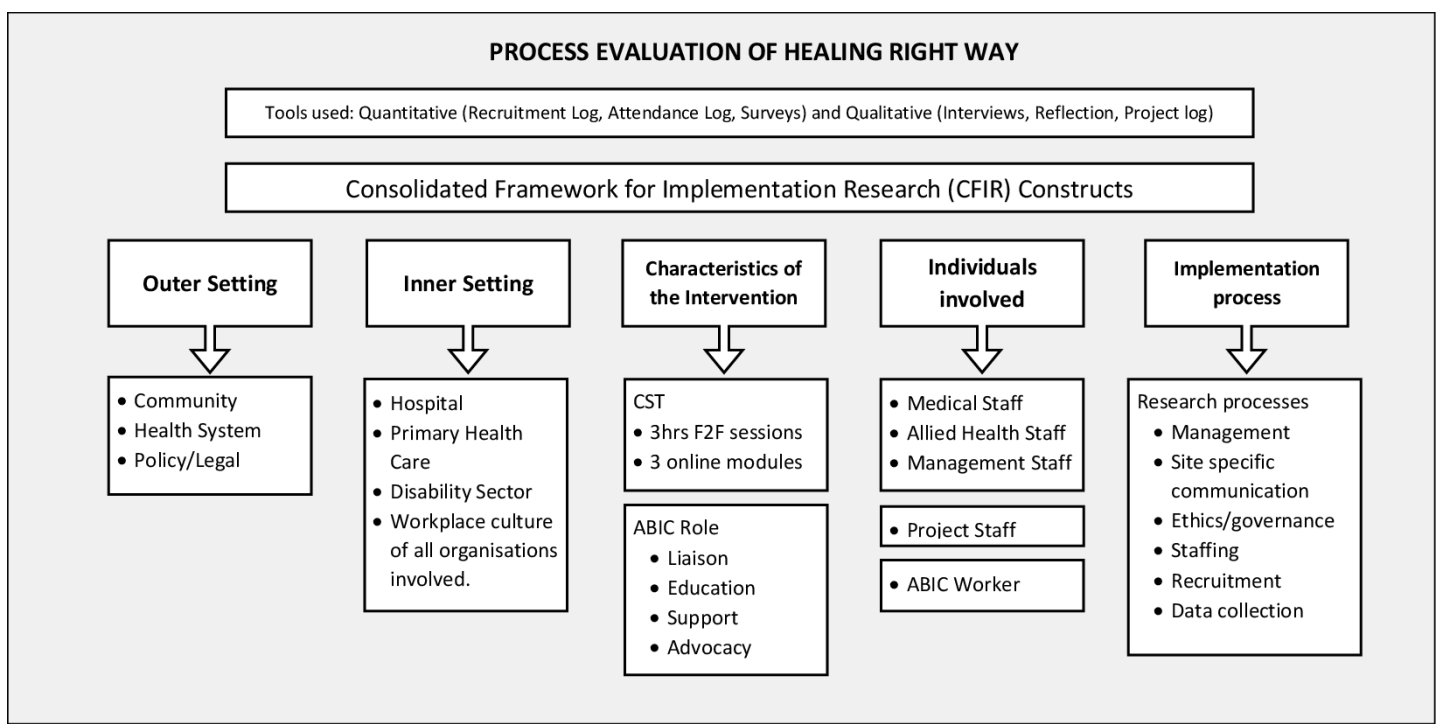

Figure 2 Healing Right Way process evaluation using the Consolidated Framework for Implementation Research. ABIC, Aboriginal Brain Injury Coordinator; CST, Cultural Security Training; F2F, Face-to-face 
and the leader of the ABIC working group who provides primary supervision and monitoring of the ABIC intervention component. The interview schedule incorporates questions that map to specific CFIR constructs; however, the questions evolve over time; for example, examining specific issues with initiating a multisite trial will be an early focus, while incorporation of lessons learnt will be a focus later. The intent is to facilitate regular feedback about barriers and enablers to implementing clinical care pathways experienced throughout the progress of HRW, ${ }^{40}$ with the feedback loop aiming to improve current and future implementation. These interviews will also consider the complexity of managing a multisite intervention with changing staff dynamics and context within and between sites, together with any planned or actual deviations from the prescribed HRW protocol along with reasons and context.

Documentation of recruitment of participants for HRW will be extracted from the screening log of the main trial to determine potential patient eligibility and enrolment in the study. Additional data relevant to participant screening will be collected from HRW operational team meeting minutes and by interviews with the chief investigator and project manager.

The complexity of data collection and participant follow-up for the trial itself (eg, statewide location of participants; the recruitment, training and retention of staff to conduct the follow-up assessments) will be another area of focus. Evaluation data will be gathered from review of project documentation, audit of data collection, project $\log$ and via the interviews with programme staff.

The collaboration between the project team and all partner organisations associated with HRW will be assessed annually via the Collaboration Health Assessment Tool $^{41}$ to gain the stakeholders' perspectives of their involvement in HRW. Data relating to HRW governance and broader stakeholder engagement will also be collected via review of project documentation, interviews with programme staff and the project log.

\section{Data from the cultural security training component}

As detailed in the HRW study protocol paper, ${ }^{28}$ the Cultural Security Training (CST) includes face-to-face (group) and online (individual) training modules.

Face-to-face training: this typically involves a local Aboriginal facilitator providing the cultural input and a non-Aboriginal facilitator providing the clinical input. An attendance $\log$ will be kept for face-to-face training at each site. Surveys collecting demographic information (eg, discipline and years of clinical experience working with patients with $\mathrm{ABI}$ ), staff satisfaction and impact of the training will be collected following the CST face-toface sessions. A member of the process evaluation team will observe and take notes of levels of participation and interaction of during the face-to-face sessions, as well as adherence to the content and timing protocol. In addition, this person will participate in a debriefing session with each facilitator after each session.
Online training: following completion of the online training component, staff will complete an additional survey relating to staff satisfaction and reflection on the CST programme overall. Web analytics data relating to participant access and completion of each module within the online training will also be collected.

Additional data on cultural security as an outcome (reflecting the effectiveness of the CST) will be collected from short surveys from HRW ABI participants rating their hospital and rehabilitation service.

\section{Data from the ABIC component}

Qualitative interviews with the trial management team, including the leader of the ABIC working group, HRW project manager and chief investigator along with document review of operations group meeting minutes will provide data concerning recruitment, training, ongoing support and retention of the ABIC role at each site. These interviews will also provide contextual information (eg, attitudes of partner organisations where the ABICs will be based and relevant community activities within each site). The ABICs' reflection and perspective on the nature of their role, including how they were trained and supported, will be gathered via interview at the end of their employment by an Aboriginal member of the evaluation team.

Quantitative data capturing activities of the ABICs relating to their role (eg, timing and nature of scheduled (required) and ad hoc contact with HRW participants, meetings attended and resources developed) will be collected via a RedCap electronic database. ABIC training completion will also be monitored and captured.

HRW ABI participants will provide feedback of their experience with the ABIC via a short questionnaire administered either face to face or by phone by an Aboriginal research assistant.

\section{Timing of data collection}

Timing of the data collection will align with HRW processes. Within each 6-month phase of the stepped wedge, the qualitative interviews with programme staff occur at specified intervals linked to that phase, beginning with chief investigator at week 4 , data operations/ leader of the ABIC working group at week 12 and project manager at week 20 of the cycle, avoiding end-of-cycle work pressures and allowing time to incorporate into next cycle. Semistructured interview schedules have been developed specific to each key informant's role, mapping directly to the CFIR constructs. The schedules have a primary focus on trial processes, current issues and solutions, and contextual changes.

Surveys from CST participants will be collected each time the training is delivered at the site (so sites 1 and 2 may receive the CST up to five times) and collected at the end of each face-to-face seminar and after completion of the online training modules. Minutes from debriefing with face-to-face CST trainers and a short questionnaire will capture their perspectives on the sessions. This is 
particularly important, given the range of presenters, with a tension between consistency of presentation and the use of localised delivery personnel.

At weeks 12 and 26 postinjury, Aboriginal ABI participants will complete brief surveys about their hospital experience, and those participants who are receiving the intervention will also complete a brief survey about their experience with the ABIC.

\section{Data analysis}

Mapping evaluation questions to CFIR

Key evaluation questions were developed for the HRW processes and for each of the components of HRW intervention (CST and ABIC). These questions provide a basis on which a systematic determination of the quality of the HRW trial can be made ${ }^{42}$ and mapped to one or more of the CFIR constructs. Table 1 highlights the questions, data sources for the question and the constructs of interest.

The evaluation questions are intended to be relevant across the life of HRW, but new questions may emerge during the trial. Should this occur, the new evaluation questions will be similarly mapped to the CFIR constructs.

\section{Data management}

Quantitative and qualitative data detailed previously will be extracted from relevant sources, for example, surveys/ RedCap, and stored electronically in password-protected folders. Data will be grouped according to the aspect of HRW to which it relates, for example, the CST, the ABIC service or the overall trial processes. Data will be organised such that it can be examined both by site and by phase of the study. Comparisons between sites and within sites will be made.

The systematic approach developed for the project log will be adopted when gathering information from appropriate meeting minutes and review of other documents. Information significant for the process evaluation will be extracted from the meeting minutes and categorised according to date, location/site and relevance to aspect of HRW intervention.

Interviews will be recorded and transcribed, with member-checking of the transcripts to enhance credibility and trustworthiness. The evaluation team will familiarise themselves with the transcripts, and using the CFIR as an initial framework, then code key issues and concepts to generate themes from the data. ${ }^{43}$

\section{Data synthesis and feedback}

Data analysis will use a range of quantitative descriptive statistical and qualitative thematic analytical methods, with triangulation and integration of results to answer the evaluation questions. Process evaluation data will be analysed both in a cross-sectional iterative manner (in parallel with HRW processes) and in a longitudinal manner throughout HRW (highlighted in figure 3). The iterative approach focuses on quality improvement approaches within HRW, where the findings directly inform the implementation of subsequent phases of HRW through identifying and addressing potential barriers/obstacles together with facilitators, thus improving intervention fidelity and implementation quality. In contrast, the longitudinal analysis is focused on supporting the interpretation of findings and identifying lessons for future implementation.

Issues, barriers and facilitators identified in the data will be weighted using the CFIR rating rules, for positive or negative influence, and strength of influence on implementation of HRW, using a five-point scale ( -2 to 2 ). This weighting will be tracked longitudinally to determine relative significance over time. For example, an issue deemed of high importance at one time-point may not remain so, while another initially thought minor may ultimately prove to be critical.

Themes generated from interview transcripts will be triangulated with HRW documentation and observation, with an initial descriptive analysis occurring for data within each phase. A cultural lens will be applied throughout the analysis with Aboriginal members of the evaluation team providing input into the interpretation of findings, ensuring that an Aboriginal world view is incorporated. Preliminary findings will be presented to the Aboriginal Reference Group for feedback. The Aboriginal and nonAboriginal members of the evaluation team will engage in reflexivity by maintaining openness, curiosity and explicitly expressing and challenging assumptions and interpretations from our various perspectives to identify incongruence, ${ }^{44}$ with any incongruence resolved via group consensus.

Data synthesis will occur according to the framework, that is, with respect to trial processes, and the HRW components of CST and ABIC. Ongoing thematic analysis will be undertaken, with subsequent quantitative data integrated into the analysis.

A reporting process has been developed for the crosssectional analysis based on the evaluation framework (figure 3), with half-yearly feedback reports provided to HRW management and HRW investigator teams to ensure issues emerging from the process evaluation monitoring are integrated into the logistics of the trial, where appropriate and possible. Reports will be staggered 3 months prior and 3 months post the intervention steps to maximise the opportunity to inform and refine the overall programme. Interim reports are intended to assist tracking progress of HRW while also providing findings from each of the main components of HRW. The report will also provide watching briefs regarding threats to implementation, key changes and recommendations for quality improvement. Findings from each interim report will be mapped to the CFIR for inclusion in the longitudinal evaluation. A collaborative process between the HRW project and evaluation teams will close the feedback loop and finalise the interim report.

To maximise utility of longitudinal evaluation findings, the Checklist for Evaluation Specific Standards will be used to develop evaluation-specific elements for reporting, ${ }^{45}$ while the Standards for Reporting Implementation 


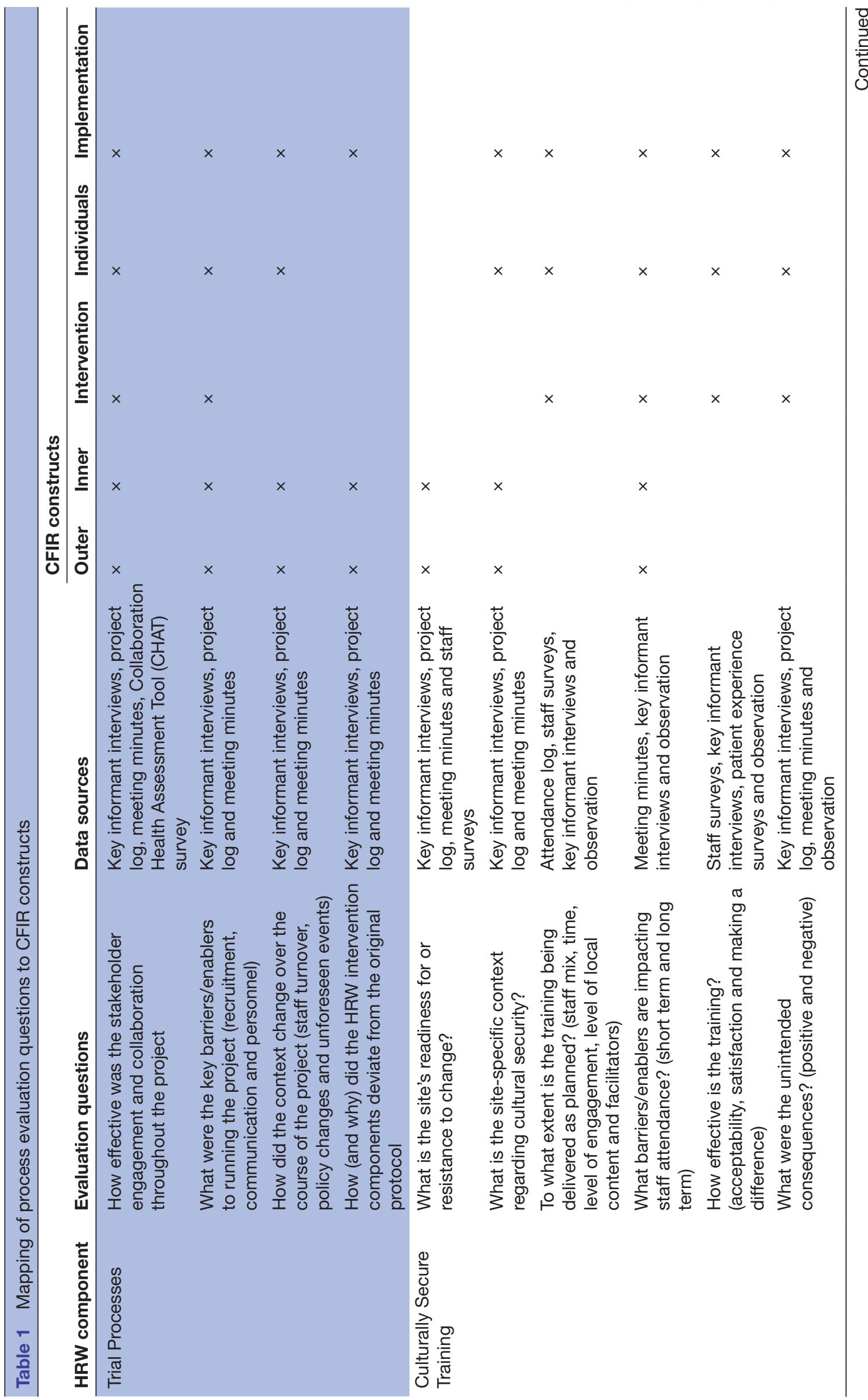




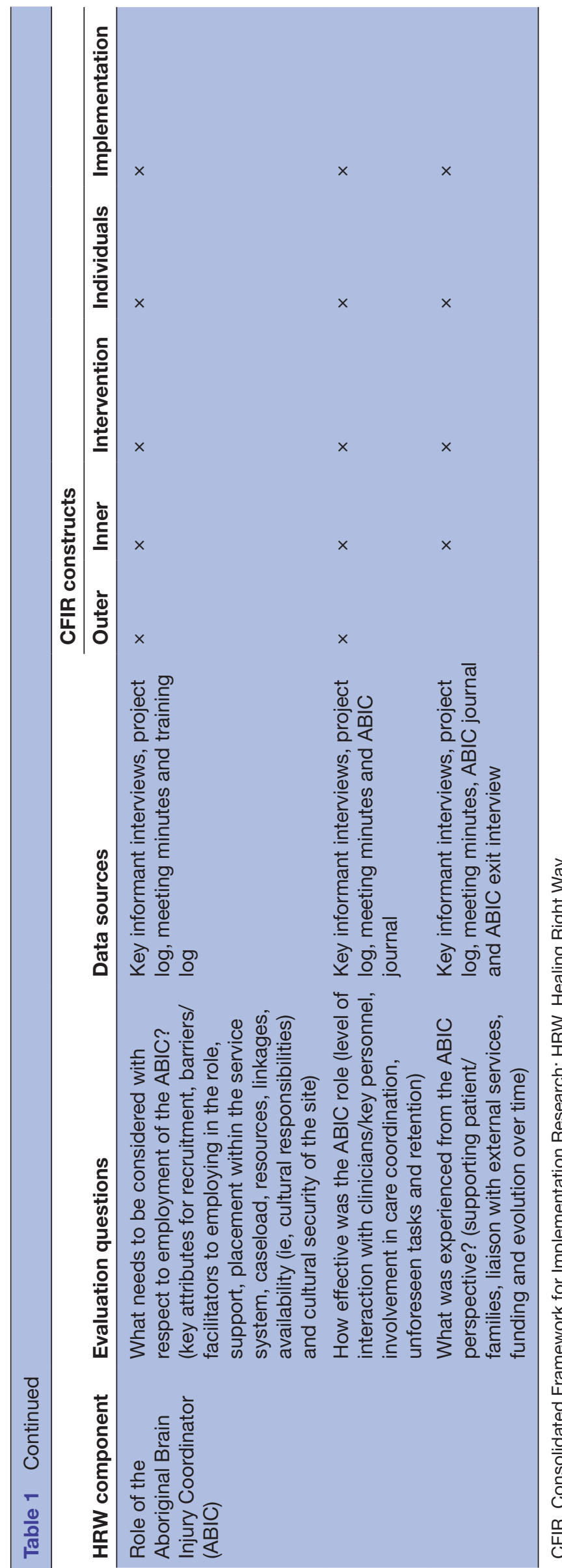




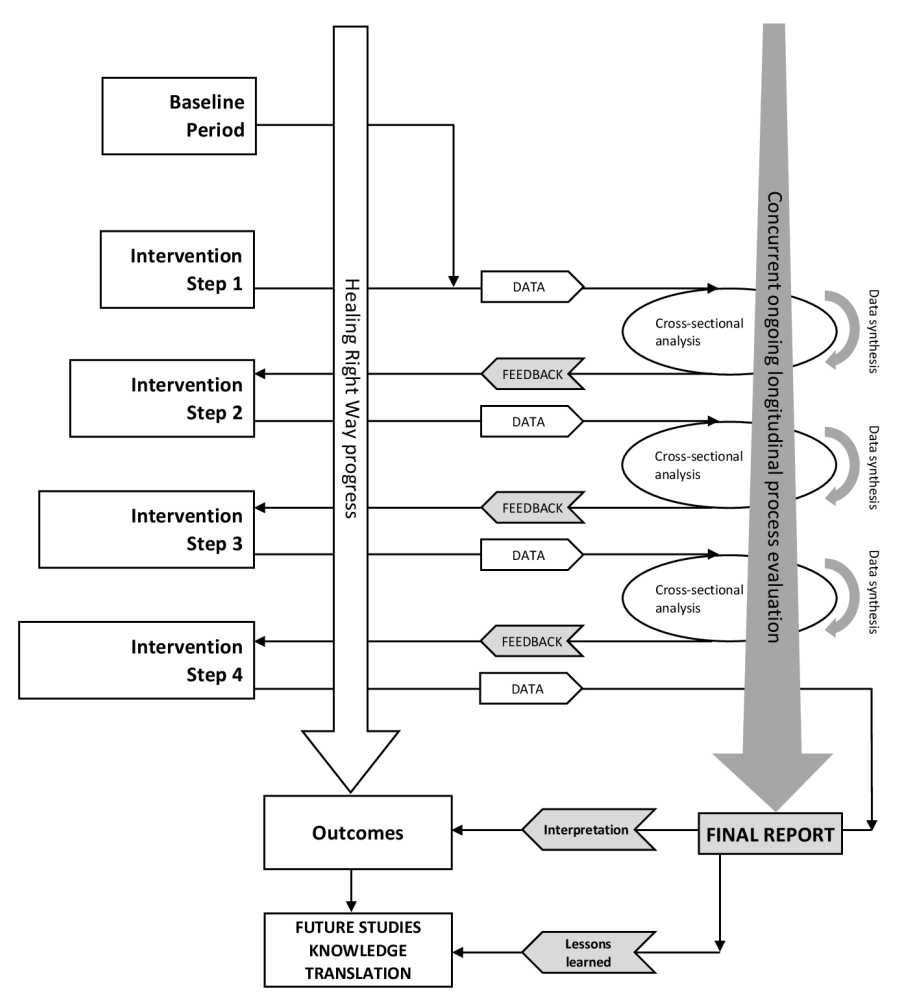

Figure 3 Synthesis and reporting plan of the HRW process evaluation. HRW, Healing Right Way.

Studies will be used to develop implementation-specific elements for reporting. ${ }^{46}$

\section{Evaluation team}

The evaluation team is led by an investigator of HRW (JMK) who will oversee evaluation progress but will not be involved with data collection or analysis. Based on the original process evaluation concept articulated in the HRW protocol, an evaluation specialist (RS) developed the evaluation design, with support from the evaluation team and three HRW investigators.

The evaluation team will work independently from the HRW team; however, an evaluation team member will be colocated within HRW to facilitate the collection of data and support the integration of findings into decisionmaking processes of HRW. ${ }^{1}$ The evaluation team will incorporate a reflexive model of inquiry into practice ${ }^{47}$ and prioritise contributions from Aboriginal members of the evaluation and HRW research teams and the HRW Aboriginal reference group to guide the evaluation synthesis.

\section{Patient and public involvement}

The evaluation will use the reference group for HRW (see box 1), including Aboriginal people who have had a brain injury. HRW's translation plan incorporates ongoing engagement with government and non-governmental organisational partners, including Aboriginal medical services and other Aboriginal consumer linkages. ${ }^{28}$
Ethics and dissemination

Multiple Aboriginal ethics protocols will be adhered to. ${ }^{48-50}$ HRW, including the process evaluation, was approved by Human Research Ethics Committees (HREC) from the hospital sites, Edith Cowan University and Western Australian Aboriginal Health Ethics Committee. Additionally, University of Western Australia HREC approved gathering of trial process data, interviews with research team managers and assessment of collaboration.

Interim and final evaluation findings will be provided to the governance committee of HRW. Findings will be disseminated via presentation at academic conferences, seminars/workshops at trial sites, regional Aboriginal health forums, peak bodies for Aboriginal health organisations, peer-reviewed journals and through the Australian Indigenous HealthinfoNet (https://healthinfonet. ecu.edu.au/). The evaluation team will be integral to the design of a translation plan outlining theory, method and practice for ensuring that the learnings and outcomes from HRW are adopted and embedded into everyday practice.

\section{DISCUSSION}

Using a cultural lens is critical for the evaluation of HRW. The team privilege Aboriginal input and ensure the evaluation is conducted according to Aboriginal values and principles. This paper highlights how to incorporate an Aboriginal perspective into a process evaluation of a complex intervention.

Evaluation has a central role in supporting and enhancing clinical research, as an evaluator can step outside the strict boundaries of the research project and consider broader settings (such as relevant policy), unanticipated events/effects and deal with controversial values and issues that may arise during the course of the parent project. Evaluation considers what standards apply, performs an empirical investigation, then synthesises the empirical findings with standards to determine merit, worth and/or value. ${ }^{38}$ Consequently, this paper focuses on how various findings from quantitative and qualitative data will be synthesised to draw meaning and come to an evaluative judgement.

There is an emerging recognition of the value of process evaluations in supporting RCTs. Assessment of the quantity and quality of the intervention delivered provides greater confidence in the interpretation of results. ${ }^{1}$ The prospective nature of the evaluation reduces potential bias of that interpretation. The concept of fidelity to trial protocol is important, ${ }^{51}$ with many contextual influences impacting on the delivery of the trial intervention. ${ }^{52}$ It can be difficult to determine which contexts matter, ${ }^{53}$ highlighting the need to consider multiple contextual factors that interact with the intervention to better understand the generalisability of the findings. ${ }^{1}$

We anticipate many contextual differences between remote, regional and urban WA communities will impact on service delivery. Given the challenges of evolving 
contexts and emerging issues, the process evaluation will be critical in supporting implementation by providing an independent analysis of 'what works' and 'lessons learnt' during the trial. ${ }^{1}$ The use of the CFIR will enable the process evaluation to deliver insights beyond the HRW project relevant to implementation of other health service interventions in Aboriginal, rural and health service contexts. The focus on implementation will potentially inform future operationalisation of culturally secure interventions for Aboriginal people after brain injury. Similarly, the learnings can inform future research using RCT designs in geographically diverse areas.

\section{Author affiliations}

${ }^{1}$ Institute for Health Research, The University of Notre Dame Australia, Fremantle, Western Australia, Australia

${ }^{2}$ Telethon Kids Institute, Perth, Western Australia, Australia

${ }^{3}$ School of Population and Global Health, University of Western Australia, Crawley,

Western Australia, Australia

${ }^{4}$ School of Medical and Health Sciences, Edith Cowan University, Joondalup, Western Australia, Australia

${ }^{5} \mathrm{Ngangk}$ Yira Research Centre for Aboriginal Health Social Equity, Murdoch University, Perth, Western Australia, Australia

${ }^{6}$ Combined Universities Centre for Rural Health, The University of Western Australia, Perth, Western Australia, Australia

${ }^{7}$ Department of Health and Medical Sciences, Edith Cowan University, Joondalup, Western Australia, Australia

Twitter Mandy J Stanley @stanley_mandy and Melanie Robinson @MelRuss12

Acknowledgements The authors acknowledge the contributions of partners and participants in their support of Healing Right Way (HRW). We acknowledge that HRW and its evaluation involve challenging experiences for participants of the trial, at times having profound impact and sadness for people, families, community and culture. We hope that the findings emanating from this evaluation can be used to allow meaning-making that can guide appropriate system change to address the needs of Aboriginal Australians who experience stroke or traumatic brain injury. The five non-Aboriginal members of the evaluation team acknowledge the critical input of the Aboriginal evaluation team member in facilitating reflexivity during the planning of the evaluation. Further Aboriginal input from our broader team, the advisory group and Aboriginal partners will be sought during the analysis and interpretation phase of the project. This will ensure that an Aboriginal lens will be cast over the data, allowing appropriate conclusions and recommendations to be made to maximise benefit and translation. Positionality statement: RS is a non-Aboriginal mixed methods health service researcher, evaluator of health and disability services, programs and policy and an advocate for people with disability and their families. MR is an Aboriginal registered nurse, educator, researcher and a director Aboriginal health who hails from the Kimberley region of Australia. She collaborates widely with Aboriginal and non-Aboriginal colleagues on research that supports Aboriginal health and well-being. She has a long-term passion for capacity building and developing the Aboriginal workforce and supporting cultural learning for non-Aboriginal staff. JW is a non-Aboriginal research officer who has worked with Aboriginal colleagues in Aboriginal health research for approximately 4 years focused on brain injury and healthy ageing. MJS is a non-Aboriginal occupational therapy educator and researcher with expertise in qualitative research methodologies and a commitment to research that improves health and well-being for people living with disability. ST is a non-Aboriginal public health physician with extensive experience working with Aboriginal people and in health services research and quality improvement. EA is a non-Aboriginal clinical speech pathologist and researcher who has worked with Aboriginal colleagues in Aboriginal health research for the last 12 years focused on brain injury and cultural security of rehabilitation services. JMK is a non-Aboriginal cardiovascular epidemiologist and mixed methods researcher with expertise in applying linked data and mixed methods to the study of Aboriginal heart disease and stroke, building capacity in Aboriginal health and using the research to advocate for changes that will improve Aboriginal health outcomes. She works closely with Aboriginal colleagues across a range of studies.
Contributors Based on the initial concept outlined in the original HRW protocol, JMK led the development of the process evaluation and obtained funding in collaboration with ST, EA and MR. RS developed the draft process evaluation protocol, prepared the draft manuscript and revised it to align with all theoretical constructs used. JW, MJS and JMK contributed to the refinement of the process evaluation protocol. JW prepared the figures. JW, MJS, MR, ST, EA and JMK contributed to the drafting of the manuscript.

Funding This work was supported by the Stroke Foundation (Australia) grant SEED1816. The parent Healing Right Way Trial is supported by National Health and Medical Research Council Grant \#1132468, Western Australian Department of Health, the Royal Perth Hospital Medical Research Foundation, the Neurological Council of Western Australia, Bega Garnbirringu Health Services, Geraldton Regional Aboriginal Medical Service, Kimberley Aboriginal Medical Services Council and the Stroke Foundation.

\section{Competing interests None declared.}

Patient and public involvement Patients and/or the public were involved in the design, or conduct, or reporting, or dissemination plans of this research. Refer to the Methods section for further details.

Patient consent for publication Not required.

Provenance and peer review Not commissioned; externally peer reviewed.

Open access This is an open access article distributed in accordance with the Creative Commons Attribution Non Commercial (CC BY-NC 4.0) license, which permits others to distribute, remix, adapt, build upon this work non-commercially, and license their derivative works on different terms, provided the original work is properly cited, appropriate credit is given, any changes made indicated, and the use is non-commercial. See: http://creativecommons.org/licenses/by-nc/4.0/.

\section{ORCID iDs}

Rachel Skoss http://orcid.org/0000-0002-7033-5063

Mandy J Stanley http://orcid.org/0000-0002-7958-5181

Elizabeth Armstrong http://orcid.org/0000-0003-4469-1117

Judith M Katzenellenbogen http://orcid.org/0000-0001-5287-5819

\section{REFERENCES}

1 Moore GF, Audrey S, Barker M, et al. Process evaluation of complex interventions: medical Research Council guidance. BMJ 2015;350:h1258.

2 Australian Bureau of Statistics. Estimates of Aboriginal and Torres Strait Islander Australians. ABS cat. No. 3238.0.55.001, 2016. Available: https://www.abs.gov.au/AUSSTATS/abs@.nsf/DetailsPage/ 3238.0.55.001June\%202016?OpenDocument

3 Australian Government. Closing the gap report, 2020. Available: https://ctgreport.niaa.gov.au/sites/default/files/pdf/closing-the-gapreport-2020.pdf

4 Katzenellenbogen JM, Sanfilippo FM, Hobbs MST, et al. Voting with their feet--predictors of discharge against medical advice in Aboriginal and non-Aboriginal ischaemic heart disease inpatients in Western Australia: an analytic study using data linkage. BMC Health Serv Res 2013;13:330.

5 Armstrong E, Coffin J, Hersh D, et al. "You felt like a prisoner in your own self, trapped": the experiences of Aboriginal people with acquired communication disorders. Disabil Rehabil 2021;43:1903-16.

6 Armstrong E, Coffin J, McAllister M, et al. 'I've got to row the boat on my own, more or less': aboriginal australian experiences of traumatic brain injury. Brain Impairment 2019;20:120-36.

7 Durey A, Thompson SC. Reducing the health disparities of Indigenous Australians: time to change focus. BMC Health Serv Res 2012;12:151.

8 Durey A, Thompson SC, Wood M. Time to bring down the twin towers in poor Aboriginal hospital care: addressing institutional racism and misunderstandings in communication. Intern Med $\mathrm{J}$ 2012;42:17-22.

9 Taylor KP, Thompson SC, Smith JS, et al. Exploring the impact of an Aboriginal health worker on hospitalised Aboriginal experiences: lessons from cardiology. Aust Health Rev 2009;33:549-57.

10 Gubhaju L, Williams R, Jones J, et al. "Cultural Security Is an On-Going Journey..." Exploring Views from Staff Members on the Quality and Cultural Security of Services for Aboriginal Families in Western Australia. Int J Environ Res Public Health 2020;17:8480.

11 Coffin J. Rising to the challenge in Aboriginal health by creating cultural security. Aboriginal and Islander Health Worker Journal 2007;31:22-4. 
12 Teoh V, Sims J, Milgrom J. Psychosocial predictors of quality of life in a sample of community-dwelling stroke survivors: a longitudinal study. Top Stroke Rehabil 2009;16:157-66.

13 Temkin NR, Corrigan JD, Dikmen SS, et al. Social functioning after traumatic brain injury. J Head Trauma Rehabil 2009;24:460-7.

14 Armstrong E, Hersh D, Katzenellenbogen JM, et al. Study Protocol: Missing Voices - Communication Difficulties after Stroke and Traumatic Brain Injury in Aboriginal Australians. Brain Impairment 2015;16:145-56.

15 Katzenellenbogen JM, Atkins ER, Thompson SC, et al. Missing voices: profile and extent of acquired communication disorders in Aboriginal and non-Aboriginal adult stroke survivors in Western Australia using linked administrative records. Int J Stroke 2016;11:103-16.

16 Katzenellenbogen JM, Atkins E, Thompson SC, et al. Missing voices: profile, extent, and 12-month outcomes of nonfatal traumatic brain injury in Aboriginal and non-Aboriginal adults in Western Australia using linked administrative records. J Head Trauma Rehabil 2018;33:412-23.

17 National Stroke Foundation. National stroke unit program: Aboriginal stroke project, 2004.

18 Esterman A, Thompson F, Fitts M, et al. Incidence of emergency department presentations for traumatic brain injury in Indigenous and non-Indigenous residents aged 15-64 over the 9-year period 2007 2015 in North Queensland, Australia. Inj Epidemiol 2018;5:40.

19 Fitts MS, Bird K, Gilroy J, et al. A qualitative study on the transition support needs of Indigenous Australians following traumatic brain injury. Brain Impairment 2019;20:137-59.

20 Stroke Foundation. Clinical guidelines for stroke management Melbourne, Australia, 2017. Available: https://strokefoundation.org. au/What-we-do/Treatment-programs/Clinical-guidelines

21 Laycock A, Walker D, Harrison N. Researching Aboriginal health: a practical guide for researchers. Melbourne: The Lowitja Institute: Australia's National Institute for Aboriginal and Torres Strait Islander Health Research, 2011.

22 Purdie N, Dudgeon P, Walker R. Working together: Aboriginal and Torres Strait Islander mental health and wellbeing principles and practice. Canberra, ACT: Australian Government, 2010.

23 Gilroy J, Donelly M, Colmar S. Conceptual framework for policy and research development with Indigenous people with disabilities. Australian Aboriginal Studies 2013;2:42.

24 Nakata M. the Cultural Interface. The Australian Journal of Indigenous Education 2007;36:7-14.

25 Gilroy J, Dew A, Lincoln M, et al. Need for an Australian Indigenous disability workforce strategy: review of the literature. Disabil Rehabil 2017;39:1664-73.

26 Gilroy J, Donelly M, Colmar S. Twelve factors that can influence the participation of Aboriginal people in disability services. Australian Indigenous Health Bulletin 2016;16.

27 Avery S. Culture is inclusion: A narrative of Aboriginal and Torres Strait Islander people with disability. In: 2018 first peoples disability network (Australia. Sydney, Australia.

28 Armstrong E, Coffin J, Hersh D. Healing right way: study protocol for a randomised control trial to enhance rehabilitation services and improve quality of life in Aboriginal Australians after brain injury. In Press.

29 Craig P, Dieppe P, Macintyre S, et al. Developing and evaluating complex interventions: the new medical Research Council guidance. BMJ 2008;337:a1655.

30 Grant A, Treweek S, Dreischulte T, et al. Process evaluations for cluster-randomised trials of complex interventions: a proposed framework for design and reporting. Trials 2013;14:15.

31 Damschroder LJ, Aron DC, Keith RE, et al. Fostering implementation of health services research findings into practice: a consolidated framework for advancing implementation science. Implement Sci 2009;4:50.

32 Linnan L, Steckler A. Process evaluation for public health interventions and research. In: Steckler A, Linnan L, eds. Process evaluation for public health interventions and research. USA: JosseyBass, 2002: 1-23.

33 Keith RE, Crosson JC, O'Malley AS, et al. Using the consolidated framework for implementation research (CFIR) to produce actionable findings: a rapid-cycle evaluation approach to improving implementation. Implement Sci 2017;12:15.

34 Sebastian S, Thomas DP, Brimblecombe J, et al. Notes from the field: applying the consolidated framework for implementation research in a qualitative evaluation of implementation of the Queensland Aboriginal and Torres Strait Islander brief intervention training program. Eval Health Prof 2021:016327872199281.

35 Haynes A, Brennan S, Carter S, et al. Protocol for the process evaluation of a complex intervention designed to increase the use of research in health policy and program organisations (the spirit study). Implementation Sci 2014;9:113.

36 Pieper MJC, Achterberg WP, van der Steen JT, et al. Implementation of a stepwise, multidisciplinary intervention for pain and challenging behaviour in dementia (STa OP!): a process evaluation. Int $\mathrm{J}$ Integr Care 2018;18:15.

37 Brady MC, Stott DJ, Norrie J, et al. Developing and evaluating the implementation of a complex intervention: using mixed methods to inform the design of a randomised controlled trial of an ora healthcare intervention after stroke. Trials 2011;12:168.

38 Wehipeihana N. Increasing cultural competence in support of Indigenous-led evaluation: a necessary step toward Indigenous-led evaluation. Canadian Journal of Program Evaluation 2019;34.

39 Oakley A, Strange V, Bonell C, et al. Process evaluation in randomised controlled trials of complex interventions. BMJ 2006;332:413-6.

40 Evans-Lacko S, Jarrett M, McCrone P, et al. Facilitators and barriers to implementing clinical care pathways. BMC Health Serv Res 2010;10:182

41 Salignac F, Marjolin A, Noone J, et al. Measuring dynamic collaborations: collaborative health assessment tool. Australian Journal of Public Administration 2019;10:227-49.

42 Scriven M. Evaluation thesaurus. 4th ed. London: Sage Publications, 1991.

43 Pope C, Ziebland S, Mays N. Qualitative research in health care. analysing qualitative data. BMJ 2000;320:114-6.

44 Poland B, Frohlich K, Haines RJ, et al. The social context of smoking: the next frontier in tobacco control? Tob Control 2006;15:59-63.

45 Montrosse-Moorhead B, Griffith JC. Toward the development of reporting standards for evaluations. Am J Eval 2017;38:577-602.

46 Pinnock H, Barwick M, Carpenter CR, et al. Standards for reporting implementation studies (STARI) statement. BMJ 2017;356:i6795.

47 van Draanen J. Introducing reflexivity to evaluation practice: an indepth case study. American Journal of Evaluation 2017;38:360-75.

48 National Health and Medical Research Council,. Ethical conduct in research with Aboriginal and Torres Strait Islander peoples and communities: guidelines for researchers and stakeholders. Commonwealth of Australia: Canberra, 2018.

49 National Health and Medical Research Council,. Keeping research on track II: a companion document to ethical conduct in research with Aboriginal and Torres Strait Islander peoples and communities: guidelines for researchers and stakeholders. Commonwealth of Australia: Canberra, 2018.

50 Australian Insitute for Aboriginal and Torres Strait Islander Studies. Guidelines for ethical research in Australian Indigenous studies, 2012

51 Wickersham K, Colbert A, Caruthers D, et al. Assessing fidelity to an intervention in a randomized controlled trial to improve medication adherence. Nurs Res 2011;60:264-9.

52 Pawson $\mathrm{R}$. The science of evaluation: a realist manifesto. London: Sage Publications, 2013.

53 Shaw J, Gray CS, Baker GR, et al. Mechanisms, contexts and points of contention: operationalizing realist-informed research for complex health interventions. BMC Med Res Methodol 2018;18:178. 